\title{
EFFICIENT FXLMS ALGORITHM WITH SIMPLIFIED SECONDARY PATH MODELS
}

\author{
Iman T. Ardekani, Hamid Sharifzadeh and Saeed Rehman
}

Computing and Information Technology Department

Unitec Institute of Technology

Auckland, New Zealans

\author{
Waleed H. Abdulla
}

\author{
ECE Department \\ The University of Auckland \\ Auckland, New Zealand
}

This paper extends the existing work on the root locus analysis of FxLMS algorithm by considering secondary path modeling errors. Rules for sketching FxLMS root locus are set out. An analytic convergence condition is then derived from the root locus plot. A deliberately-misaligned secondary path model is proposed to be used as the data preparation filter in the FxLMS algorithm. The proposed filter increases the computational efficiency of the algorithm, without changing its convergence behavior. The theoretical results are verified in practice by busing an experimental system.

Index Terms - FxLMS algorithm, root locus analysis, secondary path modeling, active noise control

\section{INTRODUCTION}

Filtered-x Least Mean Square (FxLMS) [1,2] is widely used in various adaptive signal processing applications, such as Active Noise Control (ANC) [3,4]. In these applications, there is a primary signal that propagates away from an unknown source to an error point through an unknown primary path. Also, there is a secondary (or control) signal that propagates away from a control source to the error point through a Secondary Path (SP). In this case, the signal at the error point (error signal) is the combination of the primary and secondary signals. FxLMS is responsible for the generation of the secondary signal to form a desired error signal. For this purpose, it requires a coherent reference signal and the error signal. Also, it requires a Secondary Path Model (SPM,) for preparing (or 'filtering') training data [5].

FxLMS is a simple algorithm but modeling its behavior is mathematically complicated. Hence, the existing models use various simplifying assumptions regarding the primary signal, SP or SPM [6-9]. For example, the authors have recently proposed a new framework for the analysis and design of FxLMS-based ANC systems by using Root Locus theory but they had to simplify their analysis by assuming an exact SPM $[10,11]$. The main motivation for the research conducted for this paper is to include SPM errors in the FxLMS root locus analysis. The proposed analysis has the potential of various interesting investigations, two of which are concerned by this paper. First, influence of SPM errors on the algorithm

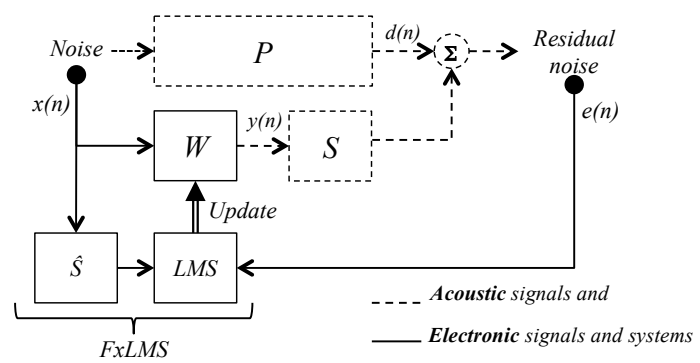

Fig. 1: General block diagram of FxLMS-based ANC

convergence is investigated. Second, misaligned SPMs that would increase efficiency of the algorithm are investigated.

\section{FXLMS ADAPTATION PROCESS}

Fig. 1 shows general diagram of FxLMS-based ANC. Noise at the error point, $d(n)$ is not measurable due to the existence of the control signal. A coherent reference signal, $x(n)$ can be measured close to the noise source. $d(n)$ is assumed to be the response of the primary path, $P$, to $x(n)$. ANC generates the control signal, $y(n)$, as the response of an adaptive filter, $W$, to $x(n) . y(n)$ propagates through the secondary path, $S$, to reach the error point, where it combines with $d(n)$ to form the residual noise $e(n)$. The FxLMS updates the $L \times 1$ weight vector of $W, \mathbf{w}(n)$, by

$$
\mathbf{w}(n+1)=\mathbf{w}(n)+\mu \mathbf{x}_{\hat{f}}(n) e(n)
$$

Here, $\mu$ is the step-size, $e(n)$ is picked up by a microphone and the filtered-reference vector, $\mathbf{x}_{\hat{f}}(n)$ can be computed by

$$
\mathbf{x}_{\hat{f}}(n)=\sum_{q} \hat{s}_{q} \mathbf{x}(n-q)
$$

where, $\mathbf{x}(n)$ is a tap vector of $x(n),\left\{\hat{s}_{q}\right\}_{0}^{Q-1}$ is an estimate of SP coefficients $\left\{s_{q}\right\}_{0}^{Q-1}$. In this paper, $\left\{s_{q}\right\}_{0}^{Q-1}$ and $\left\{\hat{s}_{q}\right\}_{0}^{Q-1}$ are referred to as SP and SPM. From the diagram,

$$
e(n)=d(n)-\sum_{q} s_{q} \mathbf{w}^{T}(n-q) \mathbf{x}(n-q)
$$


Let us define $\mathbf{z}(n)=\mathbf{F}^{T} \mathbf{x}(n)$ and $\mathbf{c}(n)=\mathbf{F}^{T}\left[\mathbf{w}(n)-\mathbf{w}_{o}\right]$, where $\mathbf{w}_{o}$ is the optimal weight vector and $\mathbf{F}$ is the modal matrix obtained by the diagonalization of $\mathbf{R}=E\left\{\mathbf{x x}^{T}\right\}$ as $\mathbf{R}=\mathbf{F} \boldsymbol{\Lambda} \mathbf{F}^{T}$. Eqs. (1-3) can be re-expressed by

$$
\begin{gathered}
\mathbf{c}(n+1)=\mathbf{c}(n)+\mu\left\{\sum_{q} \hat{s}_{q} \mathbf{z}(n-q)\right\} e(n) \\
e(n)=e_{o}(n)+\sum_{q} s_{q} \mathbf{z}^{T}(n-q) \mathbf{c}(n-q)
\end{gathered}
$$

where $e_{o}(n)$ is the optimal value of $e(n)$, that can be calculated by substituting $\mathbf{w}=\mathbf{w}_{o}$ into Eq. (3). Substituting Eq. (5) into (4) results in

$$
\begin{aligned}
& \mathbf{c}(n+1)=\mathbf{c}(n)+\mu \sum_{q} \hat{s}_{q} \mathbf{z}(n-q) e_{o}(n) \\
& +\mu \sum_{q} \hat{s}_{q} s_{q} \mathbf{z}(n-q) \mathbf{z}^{T}(n-q) \mathbf{c}(n-q)
\end{aligned}
$$

From Eq. (6), the statistical expectation of $\mathbf{c}(n)$, shown by $\overline{\mathbf{c}}(n)$ or $E\{\mathbf{c}(n)\}$, can be formulated by

$$
\begin{aligned}
& \overline{\mathbf{c}}(n+1)=\overline{\mathbf{c}}(n)+\mu \sum_{q} \hat{s}_{q} E\left\{\mathbf{z}(n-q) e_{o}(n)\right\} \\
& +\mu \sum_{q} \hat{s}_{q} s_{q} E\left\{\mathbf{z}(n-q) \mathbf{z}^{T}(n-q) \mathbf{c}(n-q)\right\}
\end{aligned}
$$

Noise is a zero-mean stationary process and independent from the optimal output; hence, $\left\{\mathbf{z}(n-q) e_{o}(n)\right\}=0$. Thus,

$$
\overline{\mathbf{c}}(n+1)=\overline{\mathbf{c}}(n)+\mu \sum_{q} \hat{s}_{q} s_{q} \boldsymbol{\Lambda} \overline{\mathbf{c}}(n-q)
$$

where $\boldsymbol{\Lambda}=E\left\{\mathbf{z z}^{T}\right\}$ is a diagonal matrix with positive real eigenvalues $\lambda_{1}, \lambda_{2}, \ldots$ and $\lambda_{L}$. The characteristic equations of Eq. (8) is formulated by

$$
1+\lambda_{l} \mu \underbrace{\frac{\sum_{q} \hat{s}_{q} s_{q} z^{Q-1-q}}{z^{Q}-z^{Q-1}}}_{\hat{H}_{F x L M S}(z)}=0
$$

Eq. (9) is in the standard form of $1+\beta H(z)=0 . \quad H(z)$ is called the open loop transfer function and its zeros and roots are called open loop zeros and poles [12].

\section{FXLMS ROOT LOCUS ANALYSIS}

Root locus analysis is a graphical method for examining how the poles of a system change with variation of a certain parameter [12]. Root locus plots $(\mathcal{R} \mathcal{L})$ can be sketched by following a set of rules. Recently, this technique is used to examine the poles of the FxLMS algorithm when $\mu$ varies; however, a simplified case with an exact SPM $\left(\hat{s}_{q}=s_{q}\right)$ is considered $[10,11]$. In the following, the important rules for sketching $\mathcal{R} \mathcal{L}$ of the FxLMS algorithm $\left(\hat{\mathcal{R}}_{\mathcal{L}_{\text {FxLMS }}}\right)$ for an arbitrary $\operatorname{SPM}\left(\hat{s}_{q} \neq s_{q}\right)$ is introduced. Fig. 2 shows three distinct examples for better understanding of these rules.

Rule 1 - Number of Branches: $\mathcal{R} \mathcal{L}$ has as many branches as there are open loop poles [12]. $\hat{H}_{F x L M S}$ has $Q$ poles; therefore, $\hat{\mathcal{R}} \mathcal{L}_{\text {FxLMS }}$ has $Q$ branches, shown by $\left\{\hat{B}_{q}\right\}_{1}^{Q}$.
Rule 2 - Starting Points: $\mathcal{R} \mathcal{L}$ begins at the open loop poles [12]. $\hat{H}_{F x L M S}$ has a single pole at $z=1$ and a repeated pole (order $Q-1$ ) at $z=0$; therefore, $\hat{\mathcal{R}} \mathcal{L}_{\mathrm{FxLMS}}$ begins at either $z=1$ or $z=0$. Let us assume that $\hat{B}_{1}$ begins at $z=1$ and $\hat{B}_{2}, \ldots, \hat{B}_{Q}$ begin at the repeated pole $z=0$.

Rule 3 - Ending Points: Branches of $\mathcal{R} \mathcal{L}$ end at either the the open loop zeros or go to infinity by approaching asymptote lines. The asymptotes intersect at the certain location on the real axis and radiate out with a certain angle [12].

Rule 4 - Real-axis Sections: $\mathcal{R} \mathcal{L}$ lies on the real axis to the left of an odd number of open loop poles and zeroes [12]. $\hat{H}_{F x L M S}$ has a single pole at $z=1$ and a repeated pole (or$\operatorname{der} Q-1)$ at $z=0$. The location of the zeros of $\hat{H}_{F x L M S}$ are unknown; therefore, determining all of the real-axis sections of $\hat{\mathcal{R}} \mathcal{L}_{\text {FxLMS }}$ is not possible. However, the real-axis sections around the critical point $z=1$ can be determined. The zeros of $\hat{H}_{F x L M S}$ are either complex conjugate or real. Since complex conjugate zeros are in pairs, they don't affect on the realaxis sections of the root locus. If there is an even number of real zeros to the right of $z=1$; then $\hat{\mathcal{R}} \mathcal{L}_{\text {FxLMS }}$ lies on the real-axis in a left neighborhood of $z=1$. This neighborhood is limited by the largest real zero between $z=0$ and $z=1$ (if there is any) or by the existing repeated pole at $z=0$ (if there is no zero between 0 and 1 ). In this case, $\hat{\mathcal{R}} \mathcal{L}_{\text {FxLMS }}$ does not lie on the real axis in a right neighborhood of $z=1$. Figs. $2 \mathrm{a}$ and $2 \mathrm{~b}$ show two examples for this case. If there is an odd number of real zeros to the right of $z=1, \hat{\mathcal{R}} \mathcal{L}_{\mathrm{FxLMS}}$ lies on the real-axis in a right neighborhood of $z=1$. This neighborhood is limited by the smallest real zero located behind $z=1$. In this case, $\hat{\mathcal{R}} \mathcal{L}_{\text {FxLMS }}$ does not lie on a left neighborhood of $z=1$. Fig. $2 \mathrm{c}$ shows an example for this case.

Rule 5 - Breakaway Points: The breakaway points are the roots of $\frac{\partial}{\partial z} \frac{1}{H(z)}=0$ [12]. $\hat{\mathcal{R}} \mathcal{L}_{\text {FxLMS }}$ might have various breakaway points, which do not necessarily affect the FxLMS convergence. Based on the same logic used in [10], the breakaway point located near $z=1$ (if any) is located at

$$
x_{B}=\frac{\hat{D}_{e q}}{\hat{D}_{e q}+1}
$$

where $\hat{D}_{e q}$ is given by

$$
\hat{D}_{e q}=\frac{\sum q s_{q} \hat{s}_{q}}{\sum s_{q} \hat{s}_{q}}
$$

For an exact $\operatorname{SPM}\left(\hat{s}_{q}=s_{q}\right), \hat{D}_{e q}$ (shown by $\left.D_{e q}\right)$ is the center of gravity of SP impulse response energy:

$$
D_{e q}=\frac{\sum q s_{q}^{2}}{\sum s_{q}^{2}}
$$

\section{THEORETICAL RESULTS AND DISCUSSION}

\subsection{Convergence Condition}

FxLMS algorithm converges as long as all of its $Q$ poles are located inside the unit circle. In $\hat{\mathcal{R}} \mathcal{L}_{\mathrm{FxLMS}}$, the poles 


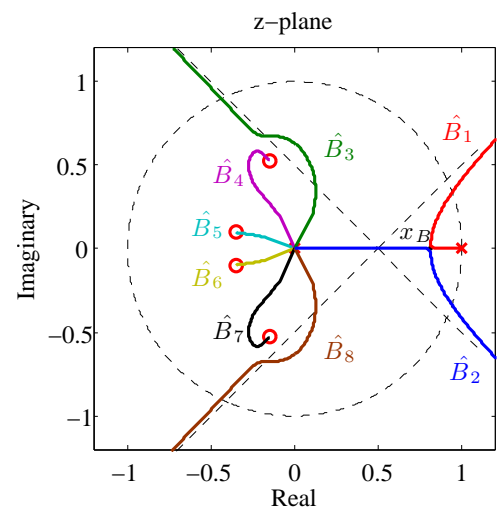

(a) Case 1: exact SPM

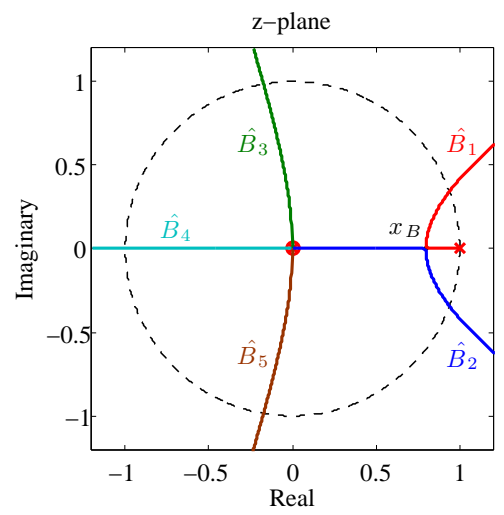

(b) Case 2: proposed SPM

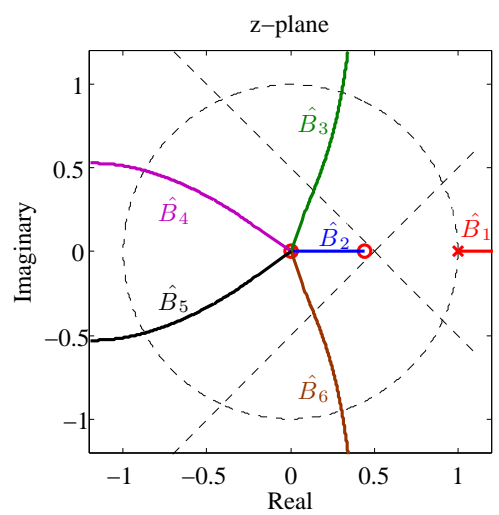

(c) Case 3: SPM leading to divergance

Fig. 2: Root locus plots of the FxLMS algorithm for SP and exact SPM: $\left\{s_{q}\right\}=\{0,0,0,1,1,0.8,0.5,-0.2\}$, proposed SPM: $\left\{\hat{s}_{q}\right\}=\{0,0,0,0,1,0,0,0\}$ and the SPM used in case 3 is $\left\{\hat{s}_{q}\right\}=\{0,0,0,-1,-1,0.8,0,0\}$

are located at the starting points when $\mu=0$. They move on $\hat{\mathcal{R}} \mathcal{L}_{\text {FxLMS }}$ when $\mu$ varies. All the poles are located either at 0 or 1 when $\mu=0$ (Rule 2). The poles moving on $\hat{B}_{2}, \ldots, \hat{B}_{Q}$ (with starting point $z=0$ ) move toward their (unknown) ending points, so they may intersect the unit circle when $\mu$ increases. However, it can be assured that they are all located inside the unit circle as long as $\mu$ is behind a certain upper-bound $\mu_{\max }$ (because they are at the center of the unit circle when $\mu=0$ ). This behaviour can be seen in the three plots of Fig. 2. There is another pole that moves on $\hat{B}_{1}$ (with starting point $z=1$ ). $\hat{B}_{1}$ lies on real-axis in either a right or left neighborhood of $z=1$ (Rule \#4). If $\hat{B}_{1}$ lies on a left neighberhood of $z=1$, then the poles moving on it can remain inside the unit circle as long as $\mu$ does not exceed a certain level; therfore the algorithm converges conditionally. Figs. $2 \mathrm{a}$ and $2 \mathrm{~b}$ show two example for the occurance of this case. If $\hat{B}_{1}$ lies on a right neighborhood of $z=1$, then the pole, moving on it, gets outside the unit circle immediately after increasing $\mu$ from zero. It means that there is no $\mu_{\max }$ behind which the algorithm converges. Fig. $2 \mathrm{c}$ shows an example for the occurance of this case.

From the above discussion, FxLMS converges only if $\hat{B}_{1}$ lies on a left neighborhood of $z=1$. This occurs when there is an odd number of zeros to the right of $z=1$ (Rule \#4). One special case occurs when there is no zeros to the right of the imaginary axis. The occurrence of this case requires all the numerator coefficients of $\hat{H}_{F x L M S}$ to be positive:

$$
\hat{s}_{q} s_{q} \geq 0
$$

This condition is very resterective but it can assure the convergence of the algorithm when the actual SP coefficients $\left\{s_{q}\right\}$ change during the operation of the algorithm. Morgan derived a similar condition for a very simplified case, when SP and SPM are pure-delay systems [2]. In this case, there are only one non-zero $s_{q}$ and one non-zero $\hat{s}_{q}$. The convergence condition given in Eq. (13) is derived for a general SP and
SPM but it includes Morgan’s convergence condition.

\subsection{Deliberately Misaligned Secondary Path Models}

Let us consider the following SPM:

$$
\hat{s}_{q}= \begin{cases}\operatorname{sign}\left(s_{q}\right) s_{r m s}, & q=\left[D_{e q}\right] \\ 0, & \text { else }\end{cases}
$$

where $\operatorname{sign}($.$) and [.] denotes the sign and nearest integer$ functions, $s_{r m s}$ denotes the root-mean-square of $\left\{s_{q}\right\}$ and $D_{e q}$ is the center of gravity of SP, given in Eq. (12). The proposed SPM has only one non-zero coefficient located at $D_{e q}$. More precisely, the energy of the proposed SPM equals to that of the actual SP but it is only concentrated at its center of gravity. The proposed SPM satisfies the convergence condition given in Eq. (13). Influences of the proposed SPM on the convergence behavior and computational efficiency of the FxLMS algorithm are investigated in the following.

\subsubsection{Convergence Behavior}

In the previous section, it is shown that the convergence of the FxLMS algorithm can be assured when $\hat{H}_{F x L M S}$ has no zeros to the right of the imaginary axis. In this case, $\hat{\mathcal{R}} \mathcal{L}_{\mathrm{FxLMS}}$ lies on the real-axis interval from 0 to 1 (Rule \#4). Both of $z=0$ and $z=1$ are starting points of the root locus; therefore, there should be a breakaway point in this interval. The location of this point is given in Eq. (10). In this situation, the pole moving on $\hat{B}_{1}$ begins at $z=1$ (for $\mu=0$ ) and moves on the real-axis when $\mu$ increases. It continues moving on the real axis until it reaches $x_{B}$ for a certain $\mu$. It leaves the real axis towards the unit circle when $\mu$ increases further. This pole is the dominant pole in $\hat{\mathcal{R}} \mathcal{L}_{\text {FxLMS }}$ because it is located closer to the unit circle, compared to the other poles. The location of $x_{B}$ dominates the FxLMS convergence behavior because the 
distance between $x_{B}$ and the critical point $z=1$ is maximal (stability margin). This behavior is previously discovered for an exact SPM [11]; however, this paper generalizes this finding for an arbitrary SPM.

For the proposed SPM, $x_{B}$ can be found by substituting Eq. (14) into (11) and (10):

$$
x_{B}=\frac{\left[D_{e q}\right]}{\left[D_{e q}\right]+1} \approx \frac{D_{e q}}{D_{e q}+1}
$$

Thus, the location of $x_{B}$ (approximately) does not change when the proposed SPM is used instead of the exact SPM. This behavior can be seen by comparing the root loci shown in Figs. $2 a$ and $2 b$. This means that the proposed SPM has no influence on the trajectory of the dominant pole in the FxLMS root locus. Consequently, it has no influence on the convergence behavior of this algorithm.

\subsubsection{Computational Efficiency}

In the case of using the proposed SPM for computing $\mathbf{x}_{\hat{f}}$ by using Eq. (2), only $L$ multiplication operations are required ( $L$ is the length of $\mathbf{x}$ ). This is while $L \times Q$ multiplication and $L \times(Q-1)$ addition operations are required in the case of using an exact SPM. This comparison shows that in the case of using the proposed SPM, computational efficiency of the FxLMS algorithm is highly increased.

\section{EXPERIMENTAL RESULTS}

For practical verification of the theoretical results, the experimental ANC system shown in Fig. 3 is used. The key component of this system is a CompctRIO controller made by National Instruments. This setup is installed in an acoustic duct with the dimentions of $1.5 \times 0.25 \times 0.25$ meter. The clock rate of the system is $40 \mathrm{MHz}$ and its operational frequency is 5 KHz. The software deployed on the CompactRIO incudes two modules: 1) SPM Identification and 2) FxLMS-based ANC. Module 1 performs the computations required for the off-line identification of a nearly-exact SPM (electro-acoustic path from the control source to the error microphone). This module generates an input white noise $(0 \mathrm{~dB}, 10-1000 \mathrm{~Hz})$ through the control source and records the output signal by using the error microphone. A model is identified by performing a simple LMS algorithm on the input and output signals [13]. The identified model has $Q=256$ coefficients. Module 2 performs the computations given in Eq. (1-2). The length of the adaptive filter $(W)$ used for this module is $L=256$ and $\mu$ is set to its optimal value [9].

In the first experiment, the identified nearly-exact SPM is used. A white noise with the power of $0 \mathrm{~dB}$ and the frequency range of $100-500 \mathrm{~Hz}$ is generated by a computer loudspeaker as the original noise entering the duct. The variation of the residual noise is shown in Fig. 4a. As seen, the original noise is attaneuated by about $20 \mathrm{~dB}$ in about $100 \mathrm{mS}$. In this experiemnt, the FxLMS-based ANC modules needs to performs

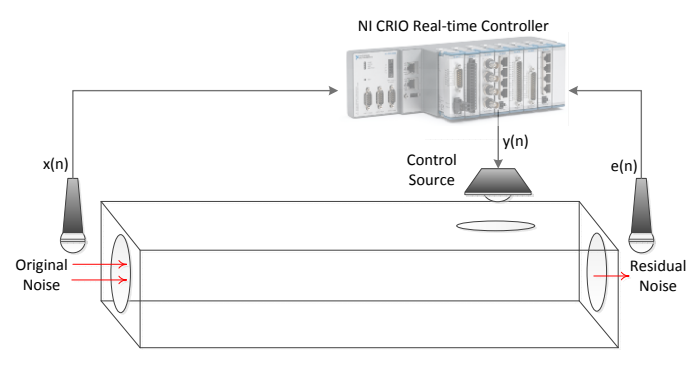

Fig. 3: Experimental FxLMS-based ANC system
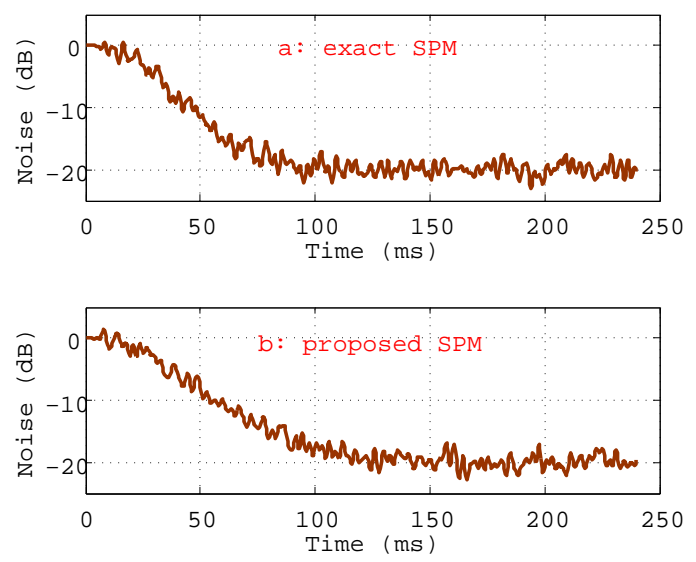

Fig. 4: Experimental results

65536 multiplication and 65280 addition operations for computing each sample of the filtered-reference vector (accodring to Section 4.2.2).

In the second experiment, the proposed SPM that has only one non-zero coefficient is used. A similar input noise is used. The frequency spectrum of the original and residual noise is shown in Fig. 4b. As seen the result is nearly identical to the result obtained from the first experiment. This is while the FxLMS-based ANC module performs only 256 multiplications and 255 addition operations in this experiment.

\section{CONCLUSION}

Root locus theory has a good potential for the analysis of FxLMS algorithm dynamics. FxLMS root locus can be sketched for an arbitrary SPM by following the rules introduced in this paper. The analysis of this root locus leads to intresting results. The first result obtained by this paper is that the FxLMS convergence can be assured if the corresponding coefficeints of SP and SPM have the same signs. The second result shows the existence of a simple SPM with only one non-zero coefficient that can maintain the FxLMS algorithm performance. This SPM can increase the computational efficiency of the FxLMS algorithm, without changing its convergence behaviour. 


\section{REFERENCES}

[1] B. Widrow, J. Glover, J. McCool, J. Kaunitz, C. Williams, R. Hearn, J. Zeidler, J. Eugene Dong, and R. Goodlin, "Adaptive noise cancelling: principles and applications," Proceedings of the IEEE, vol. 63, no. 12, pp. 1692-1716, Dec. 1975.

[2] D. R. Morgan, "An analysis of multiple correlation cancellation loops with a filter in the auxiliary path," IEEE International Conference on Acoustics, Speech, and Signal Processing, pp. 457-461, Apr. 1980.

[3] Y. Y. Kajikawa, W. W.-S. Gan, and S. M. Kuo, "Recent advances on active noise control: open issues and innovative applications," APSIPA Transactions on Signal and Information Processing, vol. 1, pp. 1-15, 112012.

[4] S. Kuo and D. Morgan, "Active noise control: a tutorial review," Proceedings of the IEEE, vol. 87, no. 6, pp. 943 - 973, 1999.

[5] J. C. Burgess, "Active adaptive sound control in a duct: computer simulation," Journal of the Acoustical Society of America, vol. 70, pp. 715-726, 1981.

[6] G. Long, F. Ling, and J. Proakis, "The LMS algorithm with delayed coefficient adaptation," IEEE Transactions on Acoustics, Speech and Signal Processing, vol. 37, no. 9, pp. 1397-1405, Sep. 1989.

[7] E. Bjarnason, "Analysis of the filtered-x LMS algorithm," IEEE Transactions on Speech and Audio Processing, vol. 3, no. 6, pp. 504-514, Nov. 1995.

[8] L. Vicente and Masgrau, "Novel FxLMS convergence condition with deterministic reference," IEEE Transactions on Signal Processing, vol. 54, no. 10, pp. 37683774, Oct. 2006.

[9] I. Tabatabaei Ardekani and W. H. Abdulla, "On the convergence of real-time active noise control systems," Signal Processing, vol. 91, no. 5, pp. 1262-1274, 2011.

[10] — "Root locus analysis and design of the adaptation process in active noise control," The Journal of the Acoustical Society of America, vol. 132, no. 4, pp. 23132324, 2012.

[11] — "Stability of residual acoustic noise variance in active control of stochastic noise," in Acoustics, Speech and Signal Processing (ICASSP), 2013 IEEE International Conference on, May 2013, pp. 379-382.

[12] K. Ogata, System Dynamics, 4th ed. Pearson/Prentice Hall, Upper Saddle River, NJ 07458, 2004, ch. 10, pp. 545-565.
[13] S. M. Kuo and D. R. Morgan, Active noise control systems: algorithms and DSP implementations. New York, NY, USA: Wiley Interscience, 1996. 